\title{
Social networks with strong spatial embedding generate non-standard epi- demic dynamics driven by higher-order clustering
}

\author{
David J. Haw ${ }^{1 a}$, Rachael Pung ${ }^{1 a}$, Jonathan M. Read ${ }^{2}$, Steven Riley ${ }^{1 *}$
}

1. MRC Centre for Outbreak Analysis and Modelling, Department of Infectious Disease Epidemiology, School of Public Health, Imperial College London, London, UK

2. Centre for Health Informatics Computing and Statistics, Lancaster Medical School, Lancaster University, Lancaster, UK

\section{${ }^{a *}$ Corresponding author. Email address s.riley@imperial.ac.uk}

\section{Abstract}

Some directly transmitted human pathogens such as influenza and measles generate sustained exponential growth in incidence, and have a high peak incidence consistent with the rapid depletion of susceptible individuals. Many do not. While a prolonged exponential phase typically arises in traditional disease-dynamic models, current quantitative descriptions of non-standard epidemic profiles are either abstract, phenomenological or rely on highly skewed offspring distributions in network models. Here, we create large socio-spatial networks to represent contact behaviour using human population density data, a previously developed fitting algorithm, and gravity-like mobility kernels. We define a basic reproductive number $R_{0}$ for this system analogous to that used for compartmental models. Controlling for $R_{0}$, we then explore networks with a household-workplace structure in which between-household contacts can be formed with varying degrees of spatial correlation, determined by a single parameter from the gravity-like kernel. By varying this single parameter and simulating epidemic spread, we are able to identify how more frequent local movement can lead to strong spatial correlation and thus induce sub-exponential outbreak dynamics with lower, later epidemic peaks. Also, the ratio of peak height to final size was much smaller when movement was highly spatially correlated. We investigate the topological properties of our networks via a generalized clustering coefficient that extends beyond immediate neighbourhoods, identifying very strong correlations between 4th order clustering and non-standard epidemic dynamics. Our results motivate the joint observation of incidence and socio-spatial human behaviour during epidemics that exhibit non-standard incidence patterns.

\section{Author Summary}

Epidemics are typically described using a standard set of mathematical models that do not capture social interactions or the way those interactions are determined by geography. Here we propose a model that can reflect social networks influenced strongly by the way people travel and we show that they lead to very different epidemic profiles. This type of model will likely be useful for forecasting. 


\section{Introduction}

Epidemics are frequently conceptualized as resulting from the transmission of a pathogen across a network. Directly transmitted pathogens propagate through susceptible human populations and create directed infection trees with an offspring-like process [15]. Each node may be a different type (e.g. children may be more infectious than adults [42]) and individuals with many contacts are more likely to cause infection than those with fewer contacts [24]. Although difficult to observe, infection trees describe a real biological process: these pathogens do not reproduce outside of a human host, so the founding pathogen population for an infectee comes directly from their infector. Further, we can conceptualise that infection trees occur when a true offspring process is constrained to pass through a social network [3, 40], with infection occurring according to a specified probability when an edge exists between a susceptible and an infectious individual.

The properties of different contact network types can be described by distributions associated with their topology [40]. First order network properties are associated with first order connections, as defined by the degree distribution. For finite random networks of reasonable size, the degree distribution is well-approximated by a Poisson in which variance is equal to the square of the mean. In contrast, for finite scale-free networks, the offspring distribution is power law-like with a much higher variance. Further, distributions of second order phenomena describe connections of length two. For example, the local clustering coefficient is a second order property, defined to be the neighbourhood density of a given node [40]. For a limited set of network types, we can use analytical expressions for higher moments of the degree distribution to calculate key properties of their potential epidemics, such as the probability of epidemic establishment and cumulative incidence $[22,28]$. Although these higher order moments are tractable for some special cases, they are seldom the primary target of theoretical studies. Semi-empirical networks that arise from detailed simulations [11] may have complex higher moments, however their impact on epidemic dynamics is obscured by the variance of their offspring distribution e.g. [25]. Here, we explicitly control our network generation algorithm so as to have non-trivial higher order structure whilst maintaining a Poisson degree distribution and a pre-specified clustering coefficient.

Epidemics can also be understood in terms of compartmental models, which are more tractable mathematically, and are equivalent to large network models with very simple topologies [35]. Key features of epidemic incidence curves is are often explained by dynamics associated with these models [1, 19]. Numerical solutions to multi-type SIR-like compartmental models are easier to obtain than for many topologies of network and can explain: the initial growth phase [30], the timing and amplitude of the peak [43], epidemic duration [21] and the total number of cases [17]. These models can efficiently describe many different types of complexity, such as age-specific susceptibility and transmissibility [16], behavioural risk groups [4] and, with increasing frequency, geographical location [34].

The basic reproductive number has been defined for both compartmental models and for network models. For compartmental models, the reproduction number is conditional on the system having a well defined period of exponential growth [18] and is defined as the average number of new infections generated by a typically infectious individual in an otherwise infectious population [18]. The word "typically" is somewhat overloaded in this definition: during the exponential phase, a system with heterogeneous population will reach a steady-state distribution of infectives, corresponding to the eigenstate of the renewal process.

For network models, the basic reproduction number is most frequently defined as the expected 
ratio of cases between the first (seed) and second generations of infection. In homogeneous networks, this is equal to the product of the average degree and the probability of transmission per link per generation. However, many studies of epidemics on networks involve high variance degree distributions [26, 25], and so this quantity must be modified to account for excess degree $[26,27]$. Here, we use $R^{*}$ to denote the expected first generation ratio if a network is homogeneous, defined to be the expected number of cases in the second generation divided by the number in the first generation. Our $R^{*}$ is therefore consistent with $\rho_{0}$ as defined in [26], although we choose not to adjust for over-dispersion, because we condition our network construction on this distribution having low variance.

The reproduction number for networks has also been defined to be more consistent with its definition for compartmental models. In [37] $R_{*}$ was defined as an asymptotic property of epidemics that were guaranteed to have an exponential phase when they occurred on infinitely large networks. We define our $R_{0}$ to be a finite-network approximation to this $R_{*}$ in [37]. This $R_{0}$ is well-defined during periods of exponential growth.

Both compartmental and network models can be embedded in space [34]. Each node can have a location in space while each compartment can refer to a single unit of space. Node density can be assigned according to known population densities and compartments can be assigned equal spatial areas but different numbers of hosts. In general, the risk of infection passing between two people decreases as the distance between their home location increases. The propensity of nodes to form links across space or for infection to spread between compartments can be quantified using mobility models borrowed from geography [12], such as the gravity and radiation models. Here, we are specifically interested in how the overall topology of a spatially-embedded network model can be driven by different movement assumptions and thus drive the gross features of the epidemics that occur on the network.

\section{Results}

We used an existing variant of the Metropolis-Hastings algorithm [35] to create a spatiallyembedded bipartite network of homes and workplaces consistent with the population density of Monrovia, Liberia, and with three illustrative movement scenarios (SI Appendix, Fig S1). An individual's propensity to choose a given workplace was determined by the distance between their home and workplace and parameters of a gravity-like kernel. The kernel was inversely proportional to distance raised to the power $\alpha$, with movement scenarios generated solely by changing the value of $\alpha$ : a control value $\alpha=0$ that removed the embedding and produced a nonspatial model; a wide kernel with $\alpha=3$ typical of developed populations [35, 38]; and a highly local kernel with $\alpha=6$ representing less developed populations (SI Appendix, Fig S1 part C compared with rural Huangshan in Ref [14]). The resulting distributions of distances from home to work were driven strongly by our choice of $\alpha$, with $95 \%$ of journeys: less than $24.12 \mathrm{~km}$ for $\alpha=0$; less than $12.91 \mathrm{~km}$ for $\alpha=3$; and less than $6.68 \mathrm{~km}$ for $\alpha=6$. Workplace links were dissolved into links between individuals in different households resulting in a network of cliques (households) that were linked according to $\alpha$.

The choice of movement kernel used to create the household-workplace networks affected gross features of simulated epidemics, even when controlling for other aspects of the network topology (Fig 1). Unipartite contact networks between households were obtained from the bipartite network of households and workplaces and were dependent on three parameters: mean household size $h$, mean number of workplace links $v$, and probability of forming a link in the workplace $p_{w}$. The mean workplace size $w$ and mean degree of the network were determined by these parameters: $w=v / p_{w}+1,\langle k\rangle=h-1+v$. Across a broad range of plausible values for 
$h, v$ and $p_{w}$, very local movement ( $\alpha=6$ ) produced later epidemics than did typical developedpopulation movement $(\alpha=3)$ or spatially random mixing ( $\alpha=0$, Fig 1A). Similarly, time to extinction was later for very local movement $(\alpha=6)$ compared with more frequent longerdistance movement $(\alpha=3)$ or the absence of spatial embedding $(\alpha=0)$. We calculate the coefficient of variation of the degree distribution $C_{V}^{2}=\left\langle k^{2}\right\rangle /\langle k\rangle-1 \sim 0.1$ for each network, independently of $\alpha$ [26].

Each simulation is assigned a value of $R^{*}$, the average number of cases in the first generation per seed infection. For moderate-to-high values of the first generation ratio $R^{*}$, there was very little difference in the final size of the outbreak for the different movement assumptions. However, for low values of $R^{*}<1.8$, the average final size of the outbreak was substantially smaller for more local kernels. This was driven by a higher probability of extinction when more local movement was assumed. The difference in final size driven by $\alpha$ was no longer present when we controlled for extinction (SI Appendix, Fig S2).

The choice of movement scenario had a substantial impact on peak incidence, even when $R^{*}$ was high and there was little difference in the final sizes (Fig 1B, Fig 2 rows 1 and 2). For example, for parameters with first generation ratios in the range $[1.8,2.2]$, average peak daily incidence as a fraction of the total population was $6.5 \times 10^{-3}$ for random spatial movement, $5.4 \times 10^{-3}$ for movement assumptions typical of developed populations and $3.0 \times 10^{-3}$ when highly local movement was assumed. The relationship between peak height and first generation ratio appeared to be strongly linear, with correlation coefficients $0.9778,0.9826$ and 0.9806 for $\alpha=0,3$ and 6 respectively.

The relationship between peak incidence and final size for the three movement scenarios illustrates further how clustering within the network directly affects gross features of an epidemic. Peak incidence is observed prior to final size during an epidemic. For the same peak height, local movement gave substantially larger final sizes. For peak daily incidences in the range $\left[3 \times 10^{-3}, 6.5 \times 10^{-3}\right]$ the final size of the outbreak was $68 \%$ when random spatial movement was assumed, $74 \%$ when movement was assumed to be typical of developed populations and $84 \%$ when highly-local movement was assumed.

For all movement scenarios, the basic reproductive number $R_{0}$ was smaller than the first generation ratio $R^{*}$ and different from the expected number of secondary cases generated by a single seed in an otherwise susceptible population. The duration of the exponential phase can be seen when incidence is plotted on a log scale: a constant gradient of log incidence is evidence of exponential growth (Figure 2, third row). However, in a network model with clearly defined generations, the generation ratio can also be used to define exponential growth: if the ratio of incidence between generation $n+1$ and $n$ is the same as the ratio between generations $n$ and $n-1$, then we can claim to have identified a period of exponential growth (Methods, Fig 2). The value of that constant observed ratio is the basic reproductive number $R_{0}$ [18].

Incidence grew exponentially for a much shorter time for highly-local movement than it did for a wider movement kernel, or for non-spatial networks, even when we controlled for $R_{0}$ to be within a narrow range (e.g. $(2,2.2]$, Fig 2$)$. Despite this being a relatively large population, there was no obvious period of exponential growth when we assumed highly local movement. Therefore, given that the basic reproductive number is defined for a genuine renewal process and its implied exponential growth [18] - it could be argued that $R_{0}$ does not exist for some of these networks for our model parameters. However, we did assign a value of $R_{0}$ for all simulations based on the most similar subset of consecutive early generations (see Methods). The amplitude of the difference was not driven in any obvious way by the underlying assumptions 
used to create the networks. These patterns were not specific to the range of values for $R_{0}$ (SI Appendix, Figs. S3, S4, S5).

Analysis of the higher-order structure of the networks suggests that movement scenarios were driving the observed characteristics of epidemics such as peak timing and attack rate via increased fourth order clustering. We use the term first order clustering for the quantity typically described as the local clustering coefficient [40]: the link density of the immediate neighbourhood of a given node. By extension, we defined order- $m$ clustering coefficient to be the expected proportion of neighbours within $m$ steps on the network who were also neighbours of each other within $m$ steps (Fig 3). We found no relationship between our assumed pattern of movement $(\alpha)$ and first or second order clustering coefficients. There was a weak relationship between $\alpha$ and third order clustering and then a very strong relationship between $\alpha$ and forth order clustering. Patterns between epidemic properties and fourth order clustering for individuals were similar to those between epidemic properties and second order clustering of households, as would be expected, given the bipartite algorithm used to create individual-level networks.

Final size increased with spatial correlation, despite peak size displaying the opposite trend for controlled $R^{*}$ or $R_{0}$. There was a strong linear relationship between order- $m$ clustering and peak size/final size, that could be explained by $\alpha$, the strength of spatial embedding, when we control for $R_{0}$ (Fig 4B). The gradient of the relationship decreased with order of clustering. Second order household clustering showed the same relationship with peak size as did fourth order individual clustering (Fig 4C). These strong linear relationships only existed when we effectively control for $R_{0}$, rather than $R^{*}$, and became less noisy when we reduced the interval used to define $R_{0}$.

We conducted a number of sensitivity analyses for these network simulation results. Analytic approximations for degree distribution $P(K=k)$ and expected first order clustering $\left\langle C C^{1}\right\rangle$ in our networks are given in Protocol S1, and are independent of $\alpha$. We confirmed these relationships in SI Appendix Figure S6 by computing these quantities on a set of networks that differ in $\alpha$. SI Appendix Figure S7 shows the relationship between $\alpha$ and clustering order 1 to 4 on networks generated using a uniform population density. SI Appendix Figure S8 shows the relationship between order- $m$ clustering $C C^{m}$ and peak size for different values of $R_{0}$. SI Appendix Figure S 9 shows clustering order 1 to 4 on networks with different $h, w$ and $p_{w}$, and SI Appendix Figure S10 provides an illustration of the relationship between higher-order clustering and rewiring probability on a commonly used network model with spatial embedding: the Watts-Strogatz Small World Network [40].

Finally, we map our network model onto a deterministic metapopulation framework so as to relate our simulations of incidence to prior analytic approximations of travelling spatial waves (Protocol S1 for analytic construction). Figure 5 shows the results of simulating on a grid of evenly spaced households of size $h=4$, where a single continuous variable describes prevalence in each household, and spatial coupling between households used in the force of infection is exactly the kernel used in the construction of our spatially embedded networks. We simulate with randomly spaced seeds (as above), and with a central seed (the center most 4 households), tracking global incidence and local time of peak incidence. The former case yields global incidence curves similar to those generated in our network model (which was seeded similarly). The latter case allows us to identify 4 distinct stages in the propagation of spatial waves that contribute to observed sub-exponential outbreak dynamics in more complex, network-based systems. SI Appendix Figure S 11 shows local peak timing in each case, and SI Appendix Figure S12 shows simulation results in 1 spatial dimension with $\alpha=6$ and $\alpha=12$, along- 
side statistical properties of prevalence, which further clarify these growth phases (c.f. figure captions for details and SI Appendix Protocol S1 for mathematical analysis).

\section{Discussion}

We have shown that non-standard epidemic dynamics can arise from strongly spatially embedded social networks. Using a flexible algorithm of assigning individuals to households and then creating a social networks with widely varying topologies, we can explain the absence of exponential growth and increased attack rate for a given peak height in terms of higher order social structure, while maintaining a standard low-variance offspring distribution. We observe consistent patterns when we control for the basic reproductive number, as measured as directly as possible from a constant ratio of incidence between generations.

The algorithm we used [35] captures the key social contexts of home and workplace while using few parameters, which has allowed us to isolate specific relationships within the epidemic dynamics, across a broad range of network topologies. However, its simplicity is a potential limitation. Specifically, an individual only belongs to a single workplace (which may represent a school or social club). In reality, people will gather non-household contacts from a variety of sources. Also, our networks are not dynamic, which may limit the generalisability of the results to short generation time pathogens.

Accurate empirical data about higher order social contacts would allow us to address some of these issues. There are a number of different approaches to gathering social contact data, including contact diaries, mobile phone apps and tag-based location tracking [31]. Diary methods and current analytical approaches can provide accurate estimates of 1st order moments (degree distribution [32]) and valuable insights into second order moments (clustering [44]). However, these data and current analytical approaches are limited for the estimation of higher order moments. It seems likely that either high resolution mobile phone location data [7] or very high coverage tag-based studies will be needed to reveal these patterns [6]. In addition, further work is needed on the use of algorithms similar to that used here to explicitly fit fully enumerated social networks to egocentric sample data from a subset of the population (or low coverage non-egocentric data) [23].

Our results can be compared with other disease-dynamic models that produce non-standard incidence profiles. Different functional forms have been suggested for the force-of-infection term in compartmental models that give polynomial growth in the early stages of an epidemic $[8,18]$. However, the key features of these model structures may be captured by a more straightforward underlying process [20]. Faster than exponential growth can be achieved with very high variance offspring distributions, which have been inferred by diary studies of social contacts [25]. There is also an extensive literature of much more abstract grid-based models of infectious disease that produce non-standard epidemic dynamic because of very local spatial processes (cellular automata [41]). We note that short periods of super-exponential growth were observed in our results for the simplified 2 dimensional metapopulation example (Fig 5B), arising from from accelerating spatial waves of incidence, not driven by the variance of the offspring distribution.

Prospective forecasting of infectious disease incidence during outbreaks [29] and seasonal epidemics [2] is an active area of public health research. Although non-mechanistic [13] and simple compartmental models $[33,39]$ have proven most reliable up to now, modern computing capacity enables studies to explore the possibility that incidence forecasts can be improved by the incorporation of realistic social network topology [36, 9]. For example, incidence of Ebola 
in west Africa in 2013-2016 and currently in central Africa exhibits strong spatial clustering and highly non-standard incidence dynamic, with short periods of exponential growth followed by low sustained peaks in incidence [10]. Future forecasting studies should explore the possibility that that sparse population density and short distances between contacts result in higher-order clustering in the social networks and the resulting non-standard incidence profiles.

\section{Methods}

\section{The Model}

We simulate 10 independent epidemics for each of 200 parameter sets $\left(h, v, p_{w}, R^{*}\right)$ drawn from a Latin hypercube, each seeded in 10 randomly selected individuals, and for each $\alpha=0,3,6$. The ranges of values used in the Latin hypercube are given in SI Appendix, Table S1, and complete parameter sets for all networks are given in SI Appendix, Table S1. Our simulations allowed us to track disease incidence and disease generation of each infection.

We simulate an epidemic on the network to reflect the natural history of Ebola, with a latent period of 9.7 days and a serial interval of 15.3 days. The generation time was calibrated by varying the relative infectiousness of a short period before the onset of symptoms. Global transmissibility $\beta$ is tuned to the value of $R^{*}$ drawn from the Latin hyper-cube. For each timestep, the probability of infection is calculated for each edge in the network. The algorithm progresses in real time with small timesteps so it can be compared with results from compartmental models. Details of the network simulation algorithm are given in [35].

\section{Assigning $R_{0}$ to each simulation}

For each simulation output, we calculate the mean reproductive ratio for each generation. For generations 1 to 9 and for each possible consecutive string of 3,4 or 5 values, we perform a linear regression fit. We define $R_{0}$ as mean reproductive ratio over the set of values for which the gradient of this fit is closest to 0 (and all values the remain larger than 1 ). This allows us to assign a value $R_{0}$ to every simulation output.

\section{Higher order clustering}

We compute our higher-order clustering coefficients on a subset of 1000 nodes in each network, chosen at random. The algorithm involves storing the network structure as lists of neighbours for each node, and performing an effective contact-tracing procedure. Though it is possible to compute these metrics for all nodes via successive multiplication of adjacency matrices, this procedure becomes computationally expensive in higher orders at networks become large.

\section{Data availability}

Results can be reproduced in the Ebola scenario in the id_spatial_sim repository [5], using scripts ebola_build.sh and ebola_run.sh.

\section{Competing Interests}

The authors declare no competing interests.

\section{Acknowledgements}

We thank Derek Cummings for useful discussions. For funding, we thank UK Medical Research Council (MRC) and the UK Department for International Development (DFID) under the MRC/DFID Concordat agreement, also part of the EDCTP2 programme supported by the European Union (UK, Centre MR/R015600/1) (DH \& SR); Wellcome Trust Investigator Award 
A

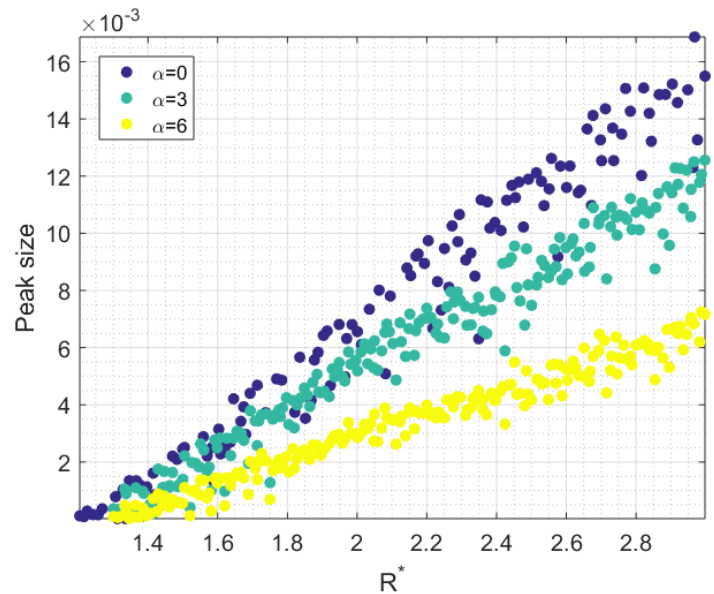

B

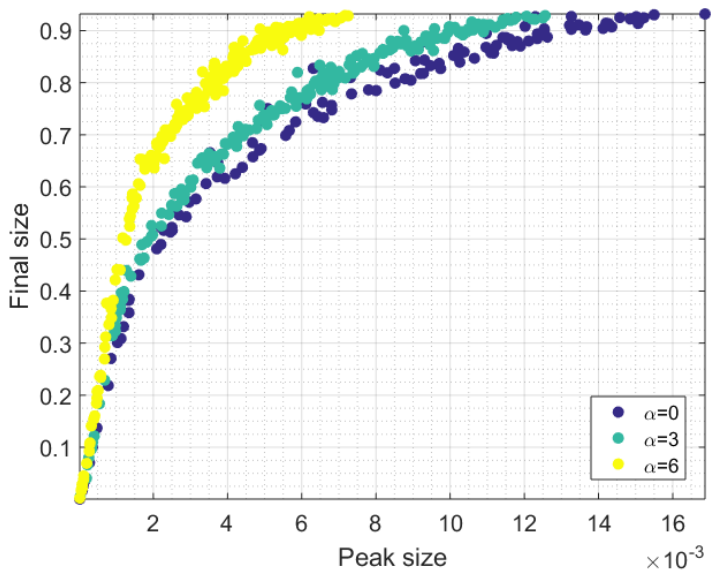

C

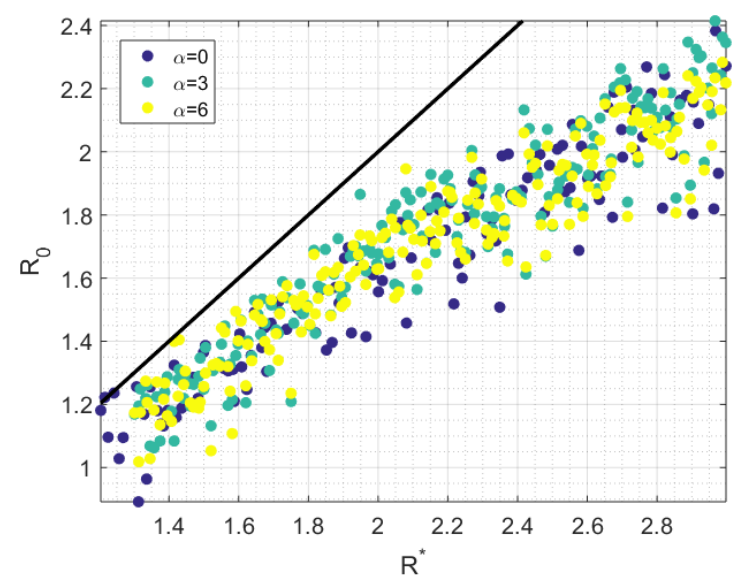

Figure 1: For each set of parameters drawn from the Latin hypercube, and for $\alpha=0,3,6$, we show relationships between: (A) $R^{*}$ and peak size, (B) peak size and final size, (C) $R^{*}$ and $R_{0}$ (with the line $R_{0}=R^{*}$ shown in black). 

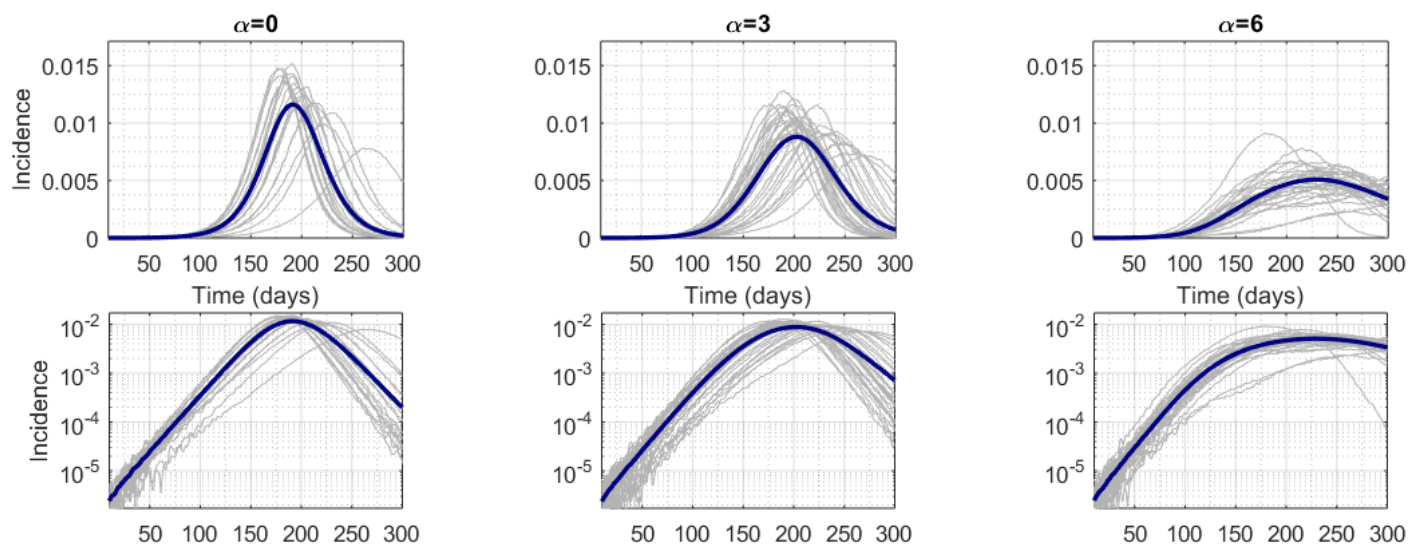

Time (days)

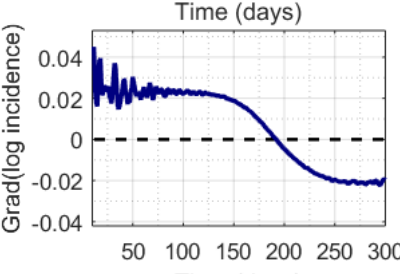

50100150200250300 Time (days)
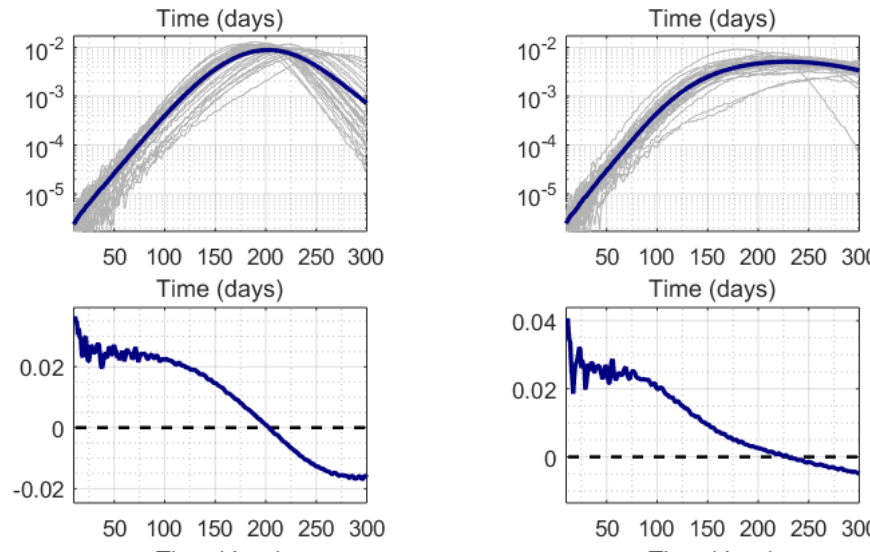

50100150200250300 Time (days)

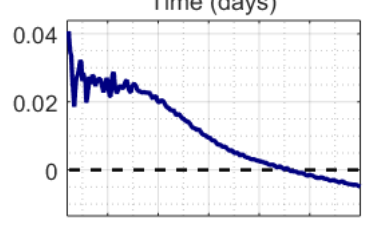

50100150200250300 Time (days)
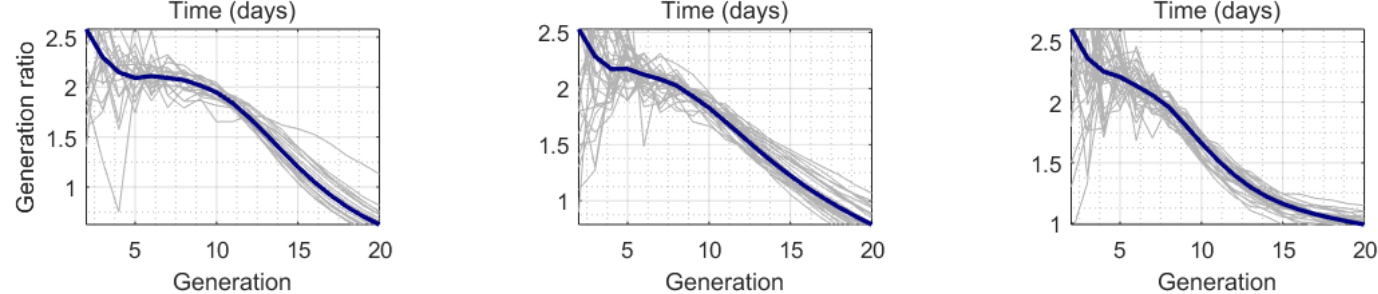

Figure 2: Columns correspond to network structures with $\alpha=0,3$ and 6 and simulations with $R_{0} \in(2,2.2]$. Exponential growth in real time is indicated by straight lines (second row) and horizontal lines (third row); horizontal lines in bottom row indicate exponential growth by generation. Figures S3 to S5 show results for a wider range of $R_{0}$ values for $\alpha=0,3,6$. National Institute for Health Research (UK, for Health Protection Research Unit funding) (SR); and the Center for Disease Control (DH). 


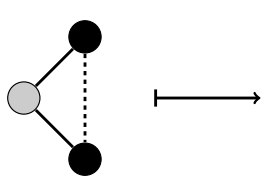

Order 1

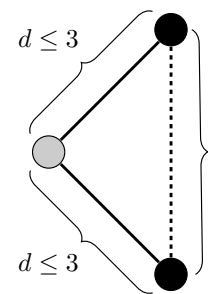

Order 3

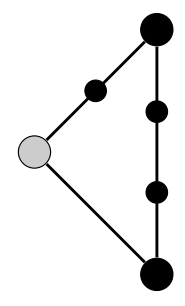

Order 3: example

Figure 3: A schematic showing the generalization of clustering coefficient $C C^{1}$ to higher orders $C C^{m}: C C_{i}^{m}$ measures the density of paths of length $d \leq m$ between the up-to- $m$ neighbours of node $i$ (where node $i$ is shown in gray). 


\section{A}

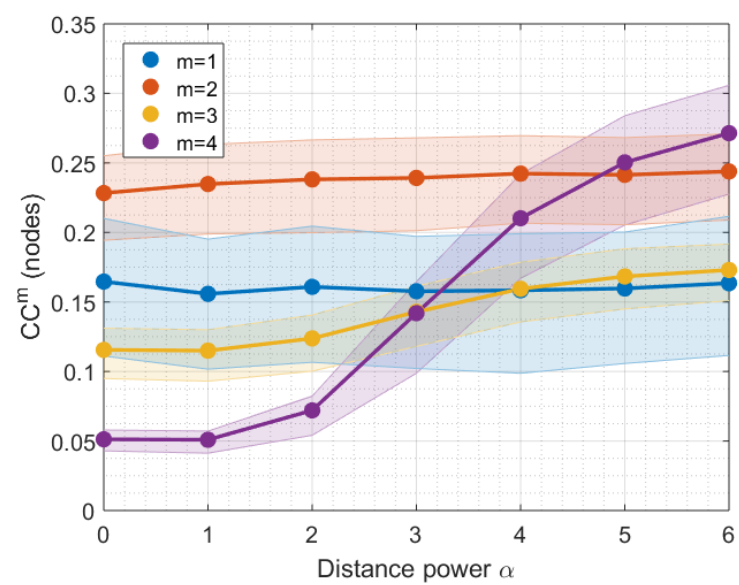

B

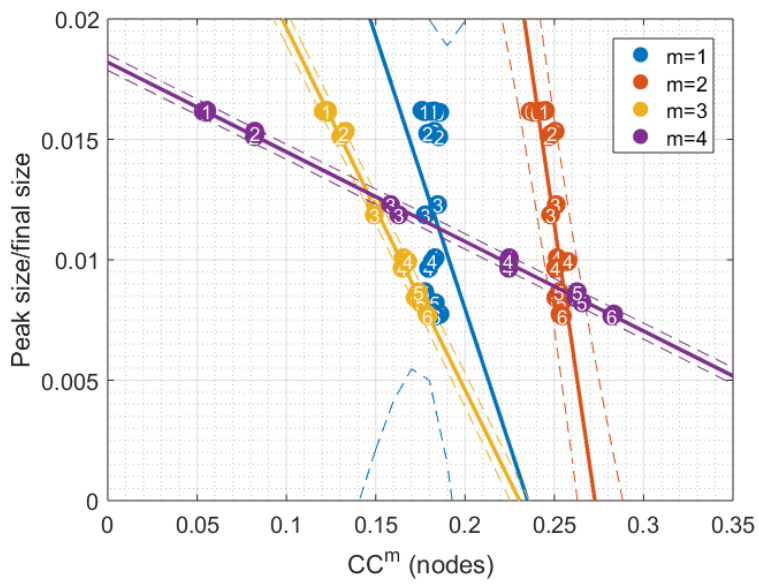

C

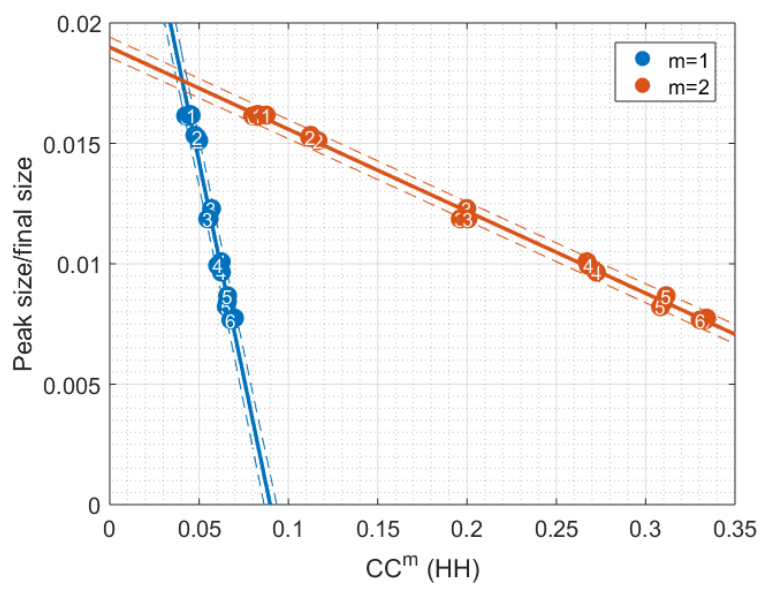

Figure 4: (A) 25-, 50- and 75-percentiles of order- $m$ clustering $C C^{m}$ on networks constructed with different values of $\alpha$ and $h=5, w=50, p_{w}=0.14,\langle k\rangle=10$ and $R_{0} \in[2,2.2)$. Plot shows mean values over 3 different networks for each parameter set; (B) Using peak size as a crude metric for sub-exponential growth (given a fixed range for $R_{0}$ ), we see linear trends emerging with higher orders of clustering. Plot shows one point per network, with 3 networks generated for each parameter set, and the mean peak size over 10 independently simulated epidemics, All points are numbered with the corresponding value of $\alpha ;(\mathbf{C})$ Similarly for the household-only networks. Solid lines show linear fits to data, and dotted lines show $95 \%$ confidence intervals. Values of linear correlation coefficient and gradient of fits are given in SI Appendix, Table S2. 
A

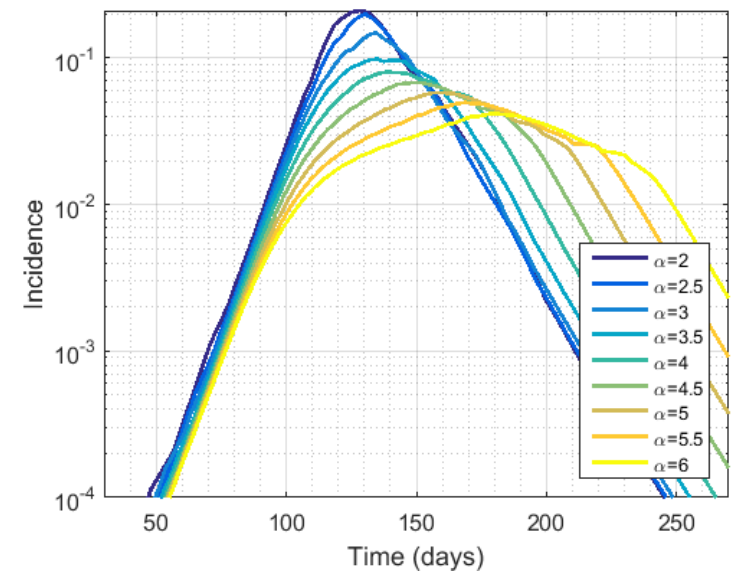

B

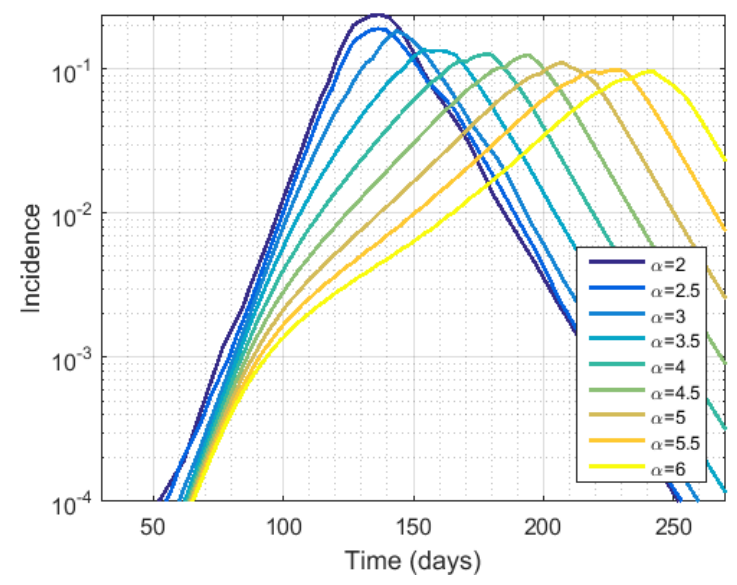

Figure 5: Mean-field approximation with $R_{0}=2.2,\langle k\rangle=10, h=4$, using a $100 \times 100$ grid of uniformly spaced households: (A) seeding in 10 randomly selected households (the same households are used in each simulation), and (B) seeding in the centre only. Incidence is given as a proportion of the total population for $\alpha$ ranging from 2 to 6 . Supplementary Figure S10 shows time of peak incidence in the case $\alpha=6$ seeded as above. 


\section{References}

[1] Roy M Anderson, Robert M May, and B Anderson. Infectious diseases of humans: dynamics and control, volume 28. Wiley Online Library, 1992.

[2] Matthew Biggerstaff, Michael Johansson, David Alper, Logan C Brooks, Prithwish Chakraborty, David C Farrow, Sangwon Hyun, Sasikiran Kandula, Craig McGowan, Naren Ramakrishnan, Roni Rosenfeld, Jeffrey Shaman, Rob Tibshirani, Ryan J Tibshirani, Alessandro Vespignani, Wan Yang, Qian Zhang, and Carrie Reed. Results from the second year of a collaborative effort to forecast influenza seasons in the United States. Epidemics, February 2018.

[3] S P Blythe, C Castillo-Chavez, J S Palmer, and M Cheng. Toward a unified theory of sexual mixing and pair formation. Math. Biosci., 107(2):379-405, December 1991.

[4] M-C Boily, C Lowndes, and M Alary. The impact of HIV epidemic phases on the effectiveness of core group interventions: insights from mathematical models. Sexually Transmitted Infections, 78(suppl 1):i78-i90, 2002.

[5] c97sr, kkokwok, dhaw, Erik Volz, and CD-DJ. c97sr/id_spatial_sim: Resubmission of subexp paper, March 2020.

[6] Ciro Cattuto, Wouter Van den Broeck, Alain Barrat, Vittoria Colizza, Jean-François Pinton, and Alessandro Vespignani. Dynamics of person-to-person interactions from distributed RFID sensor networks. PLoS One, 5(7):e11596, July 2010.

[7] Eunjoon Cho, Seth A Myers, and Jure Leskovec. Friendship and mobility: User movement in location-based social networks. In Proceedings of the 17th ACM SIGKDD International Conference on Knowledge Discovery and Data Mining, KDD '11, pages 1082-1090, New York, NY, USA, 2011. ACM.

[8] Gerardo Chowell, Lisa Sattenspiel, Shweta Bansal, and Cécile Viboud. Mathematical models to characterize early epidemic growth: A review. Physics of Life Reviews, 18:6697, 2016.

[9] John M Drake, Rajreni B Kaul, Laura W Alexander, Suzanne M O'Regan, Andrew M Kramer, J Tomlin Pulliam, Matthew J Ferrari, and Andrew W Park. Ebola cases and health system demand in Liberia. PLoS Biol., 13(1):e1002056, January 2015.

[10] Ebola Outbreak Epidemiology Team. Outbreak of Ebola virus disease in the Democratic Republic of the Congo, April-May, 2018: an epidemiological study. Lancet, 392(10143):213-221, July 2018.

[11] Stephen Eubank, Hasan Guclu, V S Anil Kumar, Madhav V Marathe, Aravind Srinivasan, Zoltán Toroczkai, and Nan Wang. Modelling disease outbreaks in realistic urban social networks. Nature, 429(6988):180-184, May 2004.

[12] Paul Expert, Tim S. Evans, Vincent D. Blondel, and Renaud Lambiotte. Uncovering spaceindependent communities in spatial networks. Proceedings of the National Academy of Sciences, 108(19):7663-7668, 2011.

[13] David C Farrow, Logan C Brooks, Sangwon Hyun, Ryan J Tibshirani, Donald S Burke, and Roni Rosenfeld. A human judgment approach to epidemiological forecasting. PLoS Comput. Biol., 13(3):e1005248, March 2017. 
[14] Tini Garske, Hongjie Yu, Zhibin Peng, Min Ye, Hang Zhou, Xiaowen Cheng, Jiabing Wu, and Neil Ferguson. Travel patterns in China. PLoS One, 6(2):e16364, February 2011.

[15] Nicholas C Grassly and Christophe Fraser. Mathematical models of infectious disease transmission. Nat. Rev. Microbiol., 6(6):477-487, May 2008.

[16] Jeff Griffiths, Dawn Lowrie, and Janet Williams. Age-structured model for the AIDS epidemic. European Journal of Operational Research, 124(1):1-14, 2000.

[17] David J Haw, Derek A T Cummings, Justin Lessler, Henrik Salje, Jonathan M Read, and Steven Riley. Differential mobility and local variation in infection attack rate, 2019.

[18] J A P Heesterbeek. A brief history of R0 and a recipe for its calculation. Acta Biotheor., 50(3):189-204, December 2001.

[19] H W Hethcote. The mathematics of infectious diseases. SIAM Rev., 42(4):599-653, October 2000.

[20] Thomas House. A general theory of early growth?: Comment on: "Mathematical models to characterize early epidemic growth: A review" by Gerardo Chowell et al. Physics of Life Reviews, 18:109-111, 2016.

[21] M. Jesse, P. Ezanno, S. Davis, and J.A.P. Heesterbeek. A fully coupled, mechanistic model for infectious disease dynamics in a metapopulation: Movement and epidemic duration. Journal of Theoretical Biology, 254(2):331 - 338, 2008.

[22] Matthew J. Keeling. The effects of local spatial structure on epidemiological invasions. Proceedings. Biological sciences / The Royal Society, 266(1421):859-67, 1999.

[23] Pavel N Krivitsky and Martina Morris. Inference for social network models from egocentrically sampled data, with application to understanding persistent racial disparities in HIV prevalence in the US. Ann. Appl. Stat., 11(1):427-455, March 2017.

[24] Kin O Kwok, Benjamin J Cowling, Vivian W I Wei, Kendra M Wu, Jonathan M Read, Justin Lessler, Derek A Cummings, J S Malik Peiris, and Steven Riley. Social contacts and the locations in which they occur as risk factors for influenza infection. Proc. Biol. Sci., 281(1789):20140709-20140709, August 2014.

[25] Quan-Hui Liu, Marco Ajelli, Alberto Aleta, Stefano Merler, Yamir Moreno, and Alessandro Vespignani. Measurability of the epidemic reproduction number in data-driven contact networks. Proceedings of the National Academy of Sciences, 115(50):12680-12685, 2018.

[26] Robert M. May and Alun L. Lloyd. Infection dynamics on scale-free networks. Phys. Rev. E, 64:066112, Nov 2001.

[27] Lauren Ancel Meyers. Contact network epidemiology: bond percolation applied to infectious disease prediction and control. Bull. Amer. Math. Soc., 44(1):63-86, 2007.

[28] Joel C Miller. Bounding the size and probability of epidemics on networks, 2008.

[29] Martha I Nelson, James O Lloyd-Smith, Lone Simonsen, Andrew Rambaut, Edward C Holmes, Gerardo Chowell, Mark A Miller, David J Spiro, Bryan Grenfell, and Cécile Viboud. Fogarty International Center collaborative networks in infectious disease modeling: Lessons learnt in research and capacity building. Epidemics, October 2018. 
[30] Hiroshi Nishiura, Gerardo Chowell, Muntaser Safan, and Carlos Castillo-Chavez. Pros and cons of estimating the reproduction number from early epidemic growth rate of influenza a (H1N1) 2009. Theor. Biol. Med. Model., 7:1, January 2010.

[31] Jonathan M Read, Ken T D Eames, and W John Edmunds. Dynamic social networks and the implications for the spread of infectious disease. J. R. Soc. Interface, 5(26):1001-1007, January 2008.

[32] Jonathan M Read, Justin Lessler, Steven Riley, Shuying Wang, Li Jiu Tan, Kin On Kwok, Yi Guan, Chao Qiang Jiang, and Derek A T Cummings. Social mixing patterns in rural and urban areas of southern China. Proceedings Of The Royal Society B-Biological Sciences, 281(1785):20140268-20140268, June 2014.

[33] Nicholas G Reich, Logan C Brooks, Spencer J Fox, Sasikiran Kandula, Craig J McGowan, Evan Moore, Dave Osthus, Evan L Ray, Abhinav Tushar, Teresa K Yamana, Matthew Biggerstaff, Michael A Johansson, Roni Rosenfeld, and Jeffrey Shaman. A collaborative multiyear, multimodel assessment of seasonal influenza forecasting in the United States. Proc. Natl. Acad. Sci. U. S. A., January 2019.

[34] Steven Riley, Ken Eames, Valerie Isham, Denis Mollison, and Pieter Trapman. Five challenges for spatial epidemic models. Epidemics, 10:68 - 71, 2015. Challenges in Modelling Infectious Disease Dynamics.

[35] Steven Riley and Neil M Ferguson. Smallpox transmission and control: spatial dynamics in Great Britain. Proc. Natl. Acad. Sci. U. S. A., 103(33):12637-12642, August 2006.

[36] Michele Tizzoni, Paolo Bajardi, Chiara Poletto, José J Ramasco, Duygu Balcan, Bruno Gonçalves, Nicola Perra, Vittoria Colizza, and Alessandro Vespignani. Real-time numerical forecast of global epidemic spreading: case study of 2009 A/H1N1pdm. BMC Med., 10(1):165, December 2012.

[37] Pieter Trapman. On analytical approaches to epidemics on networks. Theoretical Population Biology, 71(2):160 - 173, 2007.

[38] James Truscott and Neil M Ferguson. Evaluating the adequacy of gravity models as a description of human mobility for epidemic modelling. PLoS Comput. Biol., 8(10):e1002699, October 2012.

[39] Cécile Viboud, Kaiyuan Sun, Robert Gaffey, Marco Ajelli, Laura Fumanelli, Stefano Merler, Qian Zhang, Gerardo Chowell, Lone Simonsen, Alessandro Vespignani, and RAPIDD Ebola Forecasting Challenge group. The RAPIDD Ebola forecasting challenge: Synthesis and lessons learnt. Epidemics, August 2017.

[40] D J Watts and S H Strogatz. Collective dynamics of 'small-world' networks. Nature News, 393(6684):440-442, June 1998.

[41] S. Hoya White, A. Martín del Rey, and G. Rodríguez Sánchez. Modeling epidemics using cellular automata. Applied Mathematics and Computation, 186(1):193 - 202, 2007.

[42] WHO Ebola Response Team, Junerlyn Agua-Agum, Archchun Ariyarajah, Isobel M Blake, Anne Cori, Christl A Donnelly, Ilaria Dorigatti, Christopher Dye, Tim Eckmanns, Neil M Ferguson, Robert A Fowler, Christophe Fraser, Tini Garske, Wes Hinsley, Thibaut Jombart, Harriet L Mills, Srinivas Murthy, Gemma Nedjati Gilani, Pierre Nouvellet, Louise Pelletier, Steven Riley, Dirk Schumacher, Anita Shah, and Maria D Van Kerkhove. 
Ebola virus disease among children in West Africa. N. Engl. J. Med., 372(13):1274-1277, March 2015.

[43] Joseph T Wu, Steven Riley, Christophe Fraser, and Gabriel M Leung. Reducing the impact of the next influenza pandemic using household-based public health interventions. PLOS Medicine, 3(9):1-9, 082006.

[44] Xiong Xiao, Albert Jan van Hoek, Michael G Kenward, Alessia Melegaro, and Mark Jit. Clustering of contacts relevant to the spread of infectious disease. Epidemics, 17:1-9, December 2016. 